\title{
Ecologia morfofuncional de plântulas de espécies arbóreas da Estação Ecológica do Panga, Uberlândia, Minas Gerais
}

\author{
KAILA RESSEL ${ }^{1,3}$, FREDERICO A.G. GUILHERME ${ }^{1}$, IVAN SCHIAVINI ${ }^{2}$ e PAULO E. OLIVEIRA ${ }^{2}$
}

(recebido: 13 de novembro de 2002; aceito: 5 de fevereiro de 2004)

\begin{abstract}
Functional morphology and ecology of tree species seedlings of the Ecological Station of Panga, Uberlândia, Minas Gerais). The seedling functional morphology classification was used to study 122 tree species occurring at the Ecological Station of Panga, Uberlândia, Minas Gerais. The tree species of three contiguous forest formations - gallery forest, semideciduous forest and "cerradão" - had their seedlings classified according to the position, texture and cotyledon exhibition. This study intended to verify if all the studied species would fit in a functional morphology classification system and if seedling morphology would have any relation with some ecological traits, such as successional group, type of dispersal, fruiting period, seed weight, habitat, sexual system, and root/stem biomass allocation. The species were subdivided into five categories: 63 species of the phanero-epigeal-foliaceous type (PEF), 20 phanero-epigeal-reserve type (PER), 10 phanero-hypogeal-reserve type (PHR), 28 crypto-hypogeal-reserve type (CHR) and only one species of the crypto-epigeal-reserve type (CER). The classification used was very effective, representing almost all the studied species. There were no significant relationships between functional morphology groups and type of dispersal, fruiting period, root/stem biomass or micro-habitat. On the other hand, significant relationships were found between successional groups, seed weight and sexual systems with seedling functional morphology categories. Pioneer species, mostly with small and light seeds, presented almost exclusively PEF seedlings, while shade tolerant climax species, which usually produce fewer and larger seeds with plenty of reserves, showed mostly CHR seedlings. This seedling type prevails also among the dioecious species. The results demonstrated that morphology has, at least in early stages, a determinant role in the processes of tree seedling establishment and development.
\end{abstract}

Key words - cerrado, cotyledons, ecology, establishment, seedling

RESUMO - (Ecologia morfofuncional de plântulas de espécies arbóreas da Estação Ecológica do Panga, Uberlândia, Minas Gerais). Realizou-se a classificação morfofuncional de plântulas de 122 espécies arbóreas da Estação Ecológica do Panga. Espécies de três formações florestais contíguas - mata de galeria, mata mesófila semidecídua e cerradão - tiveram suas plântulas classificadas segundo suas características cotiledonares de posição, textura e exposição. O estudo objetivou verificar se as espécies estudadas enquadravam-se no sistema de classificação utilizado e se existiria alguma relação entre os tipos morfofuncionais de plântulas com grupos sucessionais, síndrome de dispersão, peso das sementes, micro-habitat, sistemas sexuais, épocas de frutificação e relação entre a biomassa de raízes e partes aéreas. As espécies foram incluídas em cinco categorias, sendo 63 espécies do tipo fanero-epígeo-foliáceo (PEF), 20 fanero-epígeo-armazenador (PER), 10 fanero-hipógeoarmazenador (PHR), 28 cripto-hipógeo-armazenador (CHR) e apenas uma espécie do tipo cripto-epígeo-armazenador (CER). A classificação morfofuncional mostrou-se eficaz, representando praticamente todas as espécies. Não houve relações significativas dos grupos morfofuncionais com a síndrome de dispersão, época de frutificação, biomassa raiz/parte aérea ou local de ocorrência das espécies estudadas. Por outro lado, foi possível relacionar os grupos sucessionais, peso das sementes e os sistemas sexuais aos grupos morfofuncionais. Espécies pioneiras, em sua maioria com sementes pequenas e leves apresentaram quase exclusivamente plântulas PEF, enquanto espécies climácicas tolerantes à sombra, usualmente com um menor número de sementes maiores e com maior quantidade de reservas, apresentaram predominantemente plântulas CHR. Este tipo também foi predominante entre espécies dióicas. Os resultados demonstraram que, pelo menos no estágio inicial, a morfologia desempenha funções determinantes nos processos de desenvolvimento e estabelecimento das plântulas de espécies arbóreas.

Palavras-chave - cerrado, cotilédones, ecologia, estabelecimento, plântulas

\section{Introdução}

A ecologia morfofuncional visa compreender as funções das estruturas morfológicas nos processos de

1. Universidade Estadual Paulista, Instituto de Biociências, Departamento de Botânica, Av. 24-A, 1515 - Bela Vista, 13506-900 Rio Claro, SP, Brasil.

2. Universidade Federal de Uberlândia, Instituto de Biologia, Caixa Postal 593, 38400-902 Uberlândia, MG, Brasil.

3. Autor para correspondência: kailarp@rc.unesp.br desenvolvimento e estabelecimento das plantas. Os principais estudos de classificação morfofuncional de plântulas foram realizadas por Duke $(1965,1969), \mathrm{Ng}$ (1978), Vogel (1980), Garwood (1983), Rousteau (1983) e Miquel (1987), com base principalmente na posição, textura, exposição e função dos cotilédones durante o processo de germinação e crescimento inicial.

As plântulas classificadas como epígeas erguem seus cotilédones acima do nível do solo. Hipógeas são as plântulas cujos cotilédones permanecem abaixo ou 
ao nível do solo, ao fim do processo de formação das plântulas. Cotilédones foliáceos são freqüentemente delgados, verdes e fotossintetizantes, enquanto cotilédones carnosos são normalmente espessos, funcionando como órgãos de reserva, podendo ou não ser fotossintetizantes (Vogel 1980). Plântulas fanerocotiledonares possuem cotilédones expostos, enquanto plântulas criptocotiledonares possuem cotilédones ocultos dentro do tegumento da semente (Duke 1965).

Baseando-se em características cotiledonares e no comprimento do hipocótilo, Miquel (1987) classificou as plântulas em: fanero-epígeo-foliáceas (PEF), faneroepígeo-armazenadoras (PER), fanero-hipógeoarmazenadoras (PHR), cripto-hipógeo-armazenadoras (CHR) e cripto-epígeo-armazenadoras (CER). Este sistema de classificação reúne os esquemas utilizados por Ng (1978) e Garwood (1983), possibilitando comparações entre os estudos realizados anteriormente com os mais recentes (Garwood 1995, Ibarra-Manríquez et al. 2001, Marques 2002) sobre a ecologia e a morfologia de plântulas em comunidades tropicais.

Visando compreender como a morfologia das plântulas está relacionada com características ecológicas das espécies arbóreas de matas do Brasil central, o presente estudo teve como objetivos: a) verificar se as espécies arbóreas estudadas enquadram-se na classificação morfofuncional proposta por Miquel (1987) e (b) verificar se existe relação entre os tipos morfofuncionais de plântulas e determinadas características ecológicas.

\section{Material e métodos}

O trabalho foi realizado na Estação Ecológica do Panga (EEP), Uberlândia, MG, pertencente à Universidade Federal de Uberlândia. A Estação ocupa uma área de 409,5 ha, (1909' - 19 $11^{\circ}$ ' S e $\left.48^{\circ} 23^{\prime}-48^{\circ} 24^{\prime} \mathrm{W}\right)$, com altitude média de $800 \mathrm{~m}$. A região caracteriza-se por um clima tropical Aw, segundo a classificação de Köppen (Rosa et al. 1991). Uma descrição da vegetação pode ser encontrada em Schiavini \& Araújo (1989).

O estudo foi realizado para 122 espécies arbóreas dentre as 162 registradas em um levantamento fitossociológico para a área (I. Schiavini e colaboradores, dados publicados parcialmente em Moreno \& Schiavini 2001). A área amostrada é representada por três formações florestais contínuas: mata de galeria, mata mesófila semidecídua e cerradão, para as quais foram demarcadas 21,150 e 40 parcelas de $100 \mathrm{~m}^{2}$, respectivamente. Todos os indivíduos com circunferência à altura do peito (CAP) acima de $15 \mathrm{~cm}$ foram registrados.

A classificação das características morfofuncionais das plântulas foi feita a partir de informações da literatura e observações diretas das plântulas, no habitat natural ou resultantes da germinação em viveiro. $\mathrm{O}$ acompanhamento da germinação foi realizado no viveiro florestal da Universidade Federal de Lavras com o objetivo de obter plântulas do maior número possível das espécies amostradas na área de estudo. Para tal, 50 sementes de 60 espécies foram semeadas em saquinhos plásticos de $17 \times 30 \mathrm{~cm}$, em um substrato composto por areia, terra e esterco (3:1:1), de forma que metade da semente ficasse exposta. Todas as sementes foram irrigadas e protegidas por sombrite de malha $50 \%$, ao longo do período de germinação.

O sistema de classificação utilizado (sensu Miquel 1987), compreende cinco tipos morfofuncionais de plântulas: faneroepígeo-foliáceo (PEF), fanero-epígeo-armazenador (PER), fanero-hipógeo-armazenador (PHR), cripto-hipógeoarmazenador (CHR) e cripto-epígeo-armazenador (CER). Os termos foram traduzidos e as abreviações tiveram como fonte o trabalho de Garwood (1995). Estas abreviações (mantidas do original em inglês para facilitar comparação) foram utilizadas no decorrer do trabalho, e representam as características dicotômicas de exposição, posição e textura: fanerocotilar $(\mathrm{P})$, criptocotilar $(\mathrm{C})$, epigeal $(\mathrm{E})$, hipogeal $(\mathrm{H})$, foliáceo $(\mathrm{F})$ e armazenador $(\mathrm{R})$.

As características morfológicas das plântulas foram associadas com outras características morfológicas e ecológicas de cada espécie, tais como: grupo sucessional, peso das sementes, sistema sexual, alocação da biomassa raiz/parte aérea, local de ocorrência, síndrome de dispersão e época de frutificação.

A caracterização das espécies arbóreas em grupos sucessionais foi feita através de observações na área de estudo. O método de classificação em grupos sucessionais foi baseado no sistema de Swaine \& Whitmore (1988), o qual consiste em três grupos principais: espécies pioneiras, clímax "exigentes de luz" e clímax "tolerantes à sombra", dependendo das exigências luminosas requeridas para o estabelecimento dos indivíduos.

O peso das sementes ( $\mathrm{S}$ ) foi obtido de sementes beneficiadas e através de informações da literatura. Para a análise, utilizaram-se três classes de peso, em gramas: $S \leq 0,1 \mathrm{~g}$; $0,1 \mathrm{~g}<\mathrm{S}<1,5 \mathrm{~g}$ e $\mathrm{S} \geq 1,5 \mathrm{~g}$.

Para a estimativa de alocação de biomassa raiz/parte aérea, foram colhidas cinco plântulas de cada espécie disponível, cinco meses após a data de emergência. As plântulas foram lavadas e divididas em parte aérea e radicular na altura do colo, secas em estufa a $70{ }^{\circ} \mathrm{C}$ por, aproximadamente, $48 \mathrm{~h}$ e pesadas até atingirem peso constante. Os peso secos foram analisados para definir a razão raiz/parte aérea.

As espécies foram separadas quanto ao local de ocorrência em: espécies de matas de galeria, mata mesófila semidecídua e cerradão.

Os tipos de dispersão foram obtidos a partir da análise da morfologia dos diásporos coletados (síndromes de dispersão) e de consultas bibliográficas. A caracterização das 
síndromes foi padronizada segundo informações de Aguiar et al. (1993), Howe \& Smallwood (1982), Howe \& Westley (1997) e Pires-O’Brien \& O’Brien (1995). Os diásporos foram classificados como zoocóricos, anemocóricos e autocóricos (estes últimos incluem as espécies barocóricas) para possibilitar a comparação dos resultados obtidos com outros estudos.

A coleta de dados fenológicos foi feita em herbários (HUFU, UB, ESAL), em fontes bibliográficas e através de observações no campo. Os dados sobre sistemas sexuais foram obtidos de Oliveira \& Paula (2001).

Para avaliar as possíveis relações da morfologia das plântulas com as características ecológicas, foram utilizadas análises de qui-quadrado de contingência. Para avaliar os resultados de alocação de biomassa em relação à morfologia das plântulas e em relação aos locais de ocorrência, foram utilizadas análises de variância (one way ANOVA; Sokal \& Rohf 1981).

\section{Resultados e Discussão}

Distribuição dos tipos morfofuncionais de plântulas - Os tipos morfofuncionais de 122 espécies arbóreas da Estação Ecológica do Panga (tabela 1) foram subdivididos em: 63 PEF, 20 PER, 10 PHR, 28 CHR e 1 CER.

A classificação morfofuncional utilizada representou de forma satisfatória todas as espécies arbóreas estudadas. Apenas Pseudobombax

Tabela 1. Espécies lenhosas de físionomias florestais da Estação Ecológica do Panga classificadas de acordo com os cinco tipos morfofuncionais (ilustrados) e seus dados ecológicos de dispersão (Dis), frutificação (Fr), grupos sucessionais (Gs), sistemas sexuais (Ss), locais de ocorrência (Lo) e classes de pesos de sementes (Ps), além dos tipos de fonte das informações obtidas (In).

Table 1. Woody species of the Panga Ecological Station grouped by their seedling functional morphology type (illustrated) and ecological data for dispersal mode (Dis), fruiting (Fr), successional group (Gs), sexual systems (Ss), occurrence (Lo), class of seed weight (Ps), and the source of information used (In).

\begin{tabular}{|c|c|c|c|c|c|c|c|c|}
\hline Família & Espécie & Dis & $\mathrm{Fr}$ & Gs & Ss & Lo & Ps & In \\
\hline$\infty$ & Faneo-epígeo-foliáceo (PEF) & & & & & & & \\
\hline Anacardiaceae & Astronium fraxinifolium Schott ex Spreng. & 2 & 1 & 2 & 3 & 5 & 1 & 2 \\
\hline Anacardiaceae & Lithrea molleoides Engl. & 1 & 1 & 2 & 1 & 7 & 1 & 3 \\
\hline Annonaceae & Unonopsis lindmanii R.E. Fr. & 1 & 2 & 3 & 1 & 4 & - & 3 \\
\hline Annonaceae & Xylopia aromatica (Lam.) Baill. & 1 & 2 & 1 & 1 & 5 & 1 & 2 \\
\hline Apocynaceae & Aspidosperma cuspa (Kunth) S.F. Blake ex Pittier & 2 & 2 & 2 & 1 & 7 & 1 & 4 \\
\hline Apocynaceae & Aspidosperma cylindrocarpon Müll. Arg. & 2 & 2 & 3 & 1 & 7 & 2 & 4 \\
\hline Apocynaceae & Aspidosperma olivaceum Müll. Arg. & 2 & 2 & 2 & 1 & 1 & - & 4 \\
\hline Apocynaceae & Aspidosperma parvifolium A. DC. & 2 & 2 & 3 & 1 & 1 & 2 & 4 \\
\hline Apocynaceae & Aspidosperma subincanum Mart. & 2 & 2 & 2 & 1 & 5 & 2 & 4 \\
\hline Bombacaceae & Pseudobombax tomentosum (Mart. \& Zucc.) Robyns & 2 & 2 & 1 & 1 & 5 & 1 & 4 \\
\hline Boraginaceae & Cordia alliodora (Ruiz \& Pav.) Oken & 2 & 2 & 1 & 1 & 1 & 1 & 2 \\
\hline Caesalpiniaceae & Bauhinia ungulata L. & 3 & 2 & 1 & 1 & 7 & - & 2 \\
\hline Caesalpiniaceae & Dimorphandra mollis Benth. & 1 & 2 & 1 & 1 & 3 & - & 1 \\
\hline Caesalpiniaceae & Senna silvestris (Vell.) H.S. Irwin \& Barneby & 3 & 2 & 1 & 1 & 7 & 1 & 4 \\
\hline Clusiaceae & Kielmeyera coriacea Mart. & 2 & 2 & 2 & 1 & 3 & 1 & 3 \\
\hline Euphorbiaceae & Phyllanthus acuminatus Vahl. & 3 & - & 2 & 2 & 1 & - & 2 \\
\hline Cecropiaceae & Cecropia pachystachya Trécul & 1 & 2 & 1 & - & 7 & 1 & 3 \\
\hline Euphorbiaceae & Croton urucurana Baill. & 1 & 2 & 1 & 2 & 4 & 1 & 1 \\
\hline Euphorbiaceae & Margaritaria nobilis L.f. & 4 & 1 & 2 & 3 & 1 & 2 & 2 \\
\hline Fabaceae & Acosmium subelegans (Mohl.) Yakovlev & 2 & 1 & 1 & 1 & 3 & 1 & 1 \\
\hline Fabaceae & Bowdichia virgilioides Kunth & 2 & 1 & 1 & 1 & 3 & 1 & 1 \\
\hline Flacourtiaceae & Casearia gossypiosperma Briq & 1 & 1 & 2 & 1 & 5 & - & 4 \\
\hline Flacourtiaceae & Casearia rupestris Eichler & 1 & 1 & 1 & 1 & 4 & 2 & 4 \\
\hline Flacourtiaceae & Casearia sylvestris $\mathrm{Sw}$. & 1 & 1 & 1 & 1 & 7 & 1 & 4 \\
\hline
\end{tabular}


continuação

\begin{tabular}{|c|c|c|c|c|c|c|c|c|}
\hline Família & Espécie & Dis & $\mathrm{Fr}$ & Gs & Ss & Lo & Ps & In \\
\hline
\end{tabular}

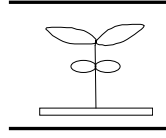

Flacourtiaceae

Lecythidaceae

Loganiaceae

Magnoliaceae

Malpighiaceae

Melastomataceae

Melastomataceae

Mimosaceae

Monimiaceae

Moraceae

Myrsinaceae

Myrsinaceae

Myrtaceae

Myrtaceae

Myrtaceae

Myrtaceae

Myrtaceae

Myrtaceae

Myrtaceae

Myrtaceae

Nyctaginaceae

Oleaceae

Polygonaceae

Rubiaceae

Rubiaceae

Rubiaceae

Rubiaceae

Sapindaceae

Sterculiaceae

Styracaceae

Styracaceae

Tiliaceae

Tiliaceae

Ulmaceae

Vochysiaceae

Vochysiaceae

Vochysiaceae

Vochysiaceae

Vochysiaceae

Faneo-epígeo-foliáceo (PEF)

Xylosma prockia (Turcz.) Turcz.

Cariniana estrellensis (Raddi) Kuntze

Strychnos pseudoquina A. St-Hil.

Talauma ovata A. St-Hil.

Byrsonima crassa Nied.

Miconia albicans (Sw.) Triana

Miconia calvescens Schrank \& Mart. ex DC.

Piptadenia gonoacantha (Mart.) J.F. Macbr.

Siparuna guianensis Aubl.

Ficus enormis (Mart. ex Miq.) Mart.

Rapanea lancifolia $\mathrm{Mez}$

Rapanea umbellata (Mart.) Mez

Calyptranthes widgreniana $\mathrm{O}$. Berg

Myrcia castrensis (O. Berg) D. Legrand

Myrcia laruotteana Cambess.

Myrcia rostrata DC.

Myrcia tomentosa (Aubl.)

Myrcia variabilis DC.

Psidium rufum DC.

Psidium sartorianum (O. Berg) Nied.

Neea hermaphrodita S. Moore

Linociera arborea Eichler

Coccoloba mollis Casar.

Alibertia sessilis (Vell.) K. Schum.

Coussarea hydrangeaefolia (Benth.) Benth. \& Hook.

Ixora gardneriana Benth.

Tocoyena formosa (Cham. \& Schltdl.) K. Schum.

Allophylus sericeus (Camb.) Radlk.

Guazuma ulmifolia Lam.

Styrax camporum Pohl

Styrax ferrugineus Nees \& Mart.

Luehea divaricata Mart.

Luehea grandiflora Mart.

Celtis iguanaea (Jacq.) Sarg.

Qualea dichotoma (Mart.) Warm.

Qualea grandiflora Mart.

Qualea multiflora Mart.

Qualea parviflora Mart.

Vochysia tucanorum Mart.

\begin{tabular}{|c|c|c|c|c|c|c|}
\hline 1 & - & 1 & - & 1 & - & 3 \\
\hline 2 & 2 & 3 & 1 & 1 & 1 & 3 \\
\hline 1 & 2 & 2 & 1 & 3 & 2 & 2 \\
\hline 1 & 2 & 3 & 1 & 2 & 2 & 3 \\
\hline 1 & 1 & 2 & 1 & 3 & - & 3 \\
\hline 1 & 1 & 1 & 1 & 3 & 1 & 3 \\
\hline 1 & 2 & 1 & 1 & 1 & - & 4 \\
\hline 2 & 2 & 2 & 1 & 1 & 1 & 3 \\
\hline 1 & 1 & 2 & 3 & 3 & - & 2 \\
\hline 1 & 2 & 2 & 2 & 3 & 1 & 2 \\
\hline 1 & 2 & 1 & 3 & 7 & - & 4 \\
\hline 1 & 1 & 2 & 3 & 5 & 1 & 3 \\
\hline 1 & - & 2 & 1 & 2 & - & 3 \\
\hline 1 & - & 2 & 1 & 2 & - & 4 \\
\hline 1 & 1 & 2 & 1 & 2 & - & 4 \\
\hline 1 & 1 & 2 & 1 & 5 & 2 & 3 \\
\hline 1 & 1 & 1 & 1 & 5 & 2 & 4 \\
\hline 1 & 1 & 2 & 1 & 3 & - & 4 \\
\hline 1 & - & 2 & 1 & 1 & 2 & 2 \\
\hline 1 & - & 2 & 1 & 1 & 2 & 2 \\
\hline 1 & 2 & 2 & 3 & 2 & 1 & 2 \\
\hline 1 & - & 2 & 1 & 4 & - & 2 \\
\hline 1 & 1 & 1 & 1 & 5 & 1 & 2 \\
\hline 1 & 1 & 1 & 3 & 7 & 1 & 3 \\
\hline 1 & 1 & 1 & 1 & 7 & 2 & 3 \\
\hline 1 & - & 3 & 1 & 2 & 1 & 4 \\
\hline 1 & - & 2 & - & 1 & - & 2 \\
\hline 1 & 1 & 1 & 2 & 1 & - & 2 \\
\hline 3 & 1 & 1 & 1 & 7 & 1 & 1 \\
\hline 1 & 2 & 2 & 1 & 7 & 2 & 3 \\
\hline 1 & 1 & 1 & 1 & 3 & 2 & 3 \\
\hline 2 & 2 & 2 & 1 & 4 & 1 & 4 \\
\hline 2 & 2 & 1 & 1 & 5 & 1 & 3 \\
\hline 1 & 1 & 2 & 2 & 1 & 2 & 2 \\
\hline 2 & 2 & 1 & 1 & 7 & 1 & 3 \\
\hline 2 & 2 & 1 & 1 & 5 & 2 & 3 \\
\hline 2 & 2 & 2 & 1 & 3 & 1 & 3 \\
\hline 2 & 2 & 2 & 1 & 3 & 1 & 4 \\
\hline 2 & 2 & 2 & 1 & 7 & 1 & 3 \\
\hline
\end{tabular}

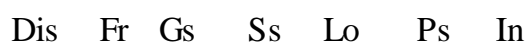

\begin{tabular}{|c|c|c|c|c|c|c|c|c|}
\hline Família & Espécie & Dis & $\mathrm{Fr}$ & Gs & Ss & Lo & Ps & In \\
\hline 0 & Faneo-epígeo-armazenador (PER) & & & & & & & \\
\hline Anacardiaceae & Tapirira guianensis Aubl. & 1 & 1 & 2 & 3 & 7 & 1 & 3 \\
\hline Bignoniaceae & Tabebuia impetiginosa (Mart. ex DC.) Standl. & 2 & 2 & 3 & 1 & 5 & 2 & 3 \\
\hline Bignoniaceae & Tabebuia roseoalba (Ridl.) Sandwith & 2 & 2 & 1 & 1 & 7 & 1 & 4 \\
\hline
\end{tabular}


continuação

\begin{tabular}{|c|c|c|c|c|c|c|c|c|}
\hline Família & Espécie & Dis & Fr & Gs & Ss & Lo & Ps & In \\
\hline ra & Faneo-epígeo-armazenador (PER) & & & & & & & \\
\hline Bignoniaceae & Tabebuia umbellata (Sond.) Sandwith & 2 & 1 & 1 & 1 & 6 & 1 & 4 \\
\hline Caesalpiniaceae & Copaifera langsdorffii Desf. & 1 & 2 & 3 & 1 & 7 & 2 & 3 \\
\hline Caesalpiniaceae & Hymenaea courbaril L. & 1 & 2 & 3 & 1 & 4 & 3 & 3 \\
\hline Erythroxylaceae & Erythroxylum deciduum A. St-Hil. & 1 & 2 & 3 & 1 & 4 & 1 & 3 \\
\hline Fabaceae & Dalbergia miscolobium Benth. & 2 & 2 & 2 & 1 & 3 & 2 & 2 \\
\hline Fabaceae & Machaerium aculeatum Raddi & 2 & 2 & 2 & 1 & 7 & 2 & 4 \\
\hline Fabaceae & Machaerium acutifolium Mart. ex Benth. & 2 & 2 & 1 & 1 & 5 & 2 & 1 \\
\hline Fabaceae & Machaerium oblongifolium Vogel & 2 & - & 2 & 1 & 1 & - & 4 \\
\hline Fabaceae & Machaerium stipitatum (DC.) Vogel & 2 & 2 & 2 & 1 & 1 & 2 & 4 \\
\hline Meliaceae & Guarea kunthiana A. Juss. & 1 & 2 & 3 & 3 & 2 & 2 & 2 \\
\hline Meliaceae & Trichilia catigua A. Juss. & 1 & 1 & 3 & 3 & 3 & - & 2 \\
\hline Meliaceae & Trichilia pallida $\mathrm{Sw}$. & 1 & 2 & 3 & 3 & 4 & 1 & 2 \\
\hline Mimosaceae & Acacia glomerosa Benth. & 3 & 2 & 1 & 1 & 5 & - & 2 \\
\hline Mimosaceae & Albizia niopoides (Spruce ex Benth.) Burkart & 3 & 1 & 1 & 1 & 1 & - & 2 \\
\hline Mimosaceae & Enterolobium gummiferum (Mart.) J.F. Macbr. & 3 & - & 2 & 1 & 3 & 2 & 2 \\
\hline Mimosaceae & Stryphnodendron polyphyllum Mart. & 1 & 2 & 2 & 1 & 3 & - & 4 \\
\hline
\end{tabular}

\begin{tabular}{|c|c|c|c|c|c|c|c|c|}
\hline Família & Espécie & Dis & Fr & Gs & Ss & Lo & Ps & In \\
\hline 8 & Faneo-(semi)hipógeo-armazenador (PER) & & & & & & & \\
\hline Ochnaceae & Ouratea castaneifolia (DC.) Engl. & 1 & 1 & 2 & 1 & 5 & 1 & 2 \\
\hline Combretaceae & Terminalia argentea (Cambess.) Mart. & 2 & 2 & 1 & 1 & 5 & 3 & 1 \\
\hline Combretaceae & Terminalia brasiliensis (Cambess. ex A. St-Hil.) Eich. & 2 & 2 & 1 & 1 & 7 & 1 & 2 \\
\hline Combretaceae & Terminalia phaeocarpa Eichler & 2 & - & 1 & 1 & 5 & - & 2 \\
\hline Connaraceae & Connarus suberosus Planch. & 1 & 1 & 2 & 1 & 3 & 2 & 3 \\
\hline Fabaceae & Ormosia fastigiata Tul. & 1 & 1 & 2 & 1 & 1 & 2 & 2 \\
\hline Mimosaceae & Anadenanthera macrocarpa (Benth.) Brenan & 3 & 2 & 2 & 1 & 7 & 2 & 3 \\
\hline Mimosaceae & Inga affinis DC. & 1 & 1 & 2 & 1 & 4 & - & 3 \\
\hline Mimosaceae & Inga fagifolia $\mathrm{G}$. Don & 1 & 2 & 3 & 1 & 4 & - & 1 \\
\hline Sapotaceae & Pouteria gardneri (Mart. \& Miq.) Baehni & 1 & 1 & 3 & 3 & 5 & 2 & 3 \\
\hline Sapotaceae & Pouteria hispida Eyma & 1 & 1 & 2 & 3 & 1 & - & 4 \\
\hline Família & Espécie & Dis & Fr & Gs & Ss & Lo & Ps & In \\
\hline
\end{tabular}

Cripto-hipógeo-armazenador (CHR)

\begin{tabular}{|c|c|c|c|c|c|c|c|c|}
\hline Annonaceae & Annona crassiflora Mart. & 1 & 1 & 3 & 1 & 3 & 1 & 1 \\
\hline Arecaceae & Acrocomia aculeata (Jacq.) Lodd. ex Mart. & 1 & 1 & 2 & - & 1 & 3 & 2 \\
\hline Arecaceae & Syagrus flexuosa (Mart.) Becc. & 1 & 2 & 2 & 2 & 3 & 3 & 3 \\
\hline Burseraceae & Protium heptaphyllum (Aubl.) Marchand & 1 & 1 & 2 & 3 & 4 & 1 & 3 \\
\hline Caryocaraceae & Caryocar brasiliense A. St-Hil. & 1 & 1 & 3 & 1 & 3 & 3 & 3 \\
\hline Chrysobalanaceae & Hirtella gracilipes (Hook. f.) Prance & 1 & 1 & 2 & 1 & 3 & - & 2 \\
\hline Clusiaceae & Calophyllum brasiliense Cambess. & 1 & 2 & 3 & 1 & 2 & 3 & 3 \\
\hline Clusiaceae & Rheedia gardneriana Planch. \& Triana & 1 & 1 & 3 & 3 & 1 & 3 & 2 \\
\hline Fabaceae & Platypodium elegans Vogel & 2 & 2 & 2 & 1 & 7 & 2 & 3 \\
\hline Hippocrateaceae & Cheiloclinium cognatum (Miers) A.C. Sm. & 1 & 2 & 2 & 1 & 4 & - & 3 \\
\hline
\end{tabular}


continuação

\begin{tabular}{|c|c|c|c|c|c|c|c|c|}
\hline Família & Espécie & Dis & Fr & Gs & Ss & Lo & Ps & In \\
\hline & Cripto-hipógeo-armazenador (CHR) & & & & & & & \\
\hline Lauraceae & Aniba heringeri Vattimo & 1 & 2 & 2 & - & 2 & 1 & 2 \\
\hline Lauraceae & Endlicheria paniculata (Spreng.) J.F. Macbr. & 1 & 2 & 3 & 3 & 4 & 2 & 2 \\
\hline Lauraceae & Nectandra cissiflora Nees & 1 & 1 & 3 & - & 4 & 2 & 4 \\
\hline Lauraceae & Ocotea corymbosa (Meisn.) Mez & 1 & 2 & 2 & 3 & 7 & 2 & 4 \\
\hline Lauraceae & Ocotea minarum (Nees \& Mart.) Mez & 1 & 1 & 2 & 3 & 5 & 2 & 4 \\
\hline Lauraceae & Ocotea percoriacea (Meisn.) Kosterm. & 1 & - & 2 & 3 & 2 & - & 4 \\
\hline Lauraceae & Ocotea pulchella Mart. & 1 & 2 & 2 & 3 & 7 & 2 & 4 \\
\hline Moraceae & Brosimum gaudichaudii Trécul & 1 & 1 & 3 & 2 & 5 & 3 & 3 \\
\hline Myrtaceae & Eugenia aurata $\mathrm{O}$. Berg & 1 & 1 & 2 & - & 3 & - & 4 \\
\hline Myrtaceae & Eugenia florida DC. & 1 & 1 & 3 & 1 & 7 & 2 & 4 \\
\hline Myrtaceae & Eugenia involucrata DC. & 1 & 1 & 3 & 1 & 1 & 2 & 4 \\
\hline Myrtaceae & Eugenia ligustrina Kiaersk. & 1 & - & 3 & 1 & 4 & - & 4 \\
\hline Rosaceae & Prunus sellowii Koehne & 1 & 2 & 2 & 1 & 3 & 2 & 2 \\
\hline Sapindaceae & Cupania vernalis Cambess. & 1 & 1 & 2 & 2 & 5 & 2 & 3 \\
\hline Sapindaceae & Matayba elaeagnoides Radlk. & 1 & 1 & 2 & 2 & 7 & 2 & 4 \\
\hline Sapindaceae & Matayba guianensis Aubl. & 1 & 1 & 1 & 2 & 7 & - & 3 \\
\hline Simaroubaceae & Picramnia sellowii Planch. & 1 & 2 & 1 & 3 & 2 & - & 3 \\
\hline Verbenaceae & Aegiphila sellowiana Cham. & 1 & 1 & 1 & 3 & 1 & 1 & 1 \\
\hline Família & Espécie & Dis & Fr & Gs & Ss & Lo & Ps & In \\
\hline
\end{tabular}

Cripto-epígeo-armazenador (CHR)

\begin{tabular}{llllllll}
\hline Myristicaceae & Virola sebifera Aubl. & 1 & 2 & 1 & 3 & 5 & 2 \\
\hline
\end{tabular}

Dis/dispersão: (1) zoocórica, (2) anemocórica, (3) autocórica; Fr/frutificação: (1) chuvosa e (2) seca; Gs/grupos sucessionais: (1) pioneira, (2) clímax exigente de luz e (3) clímax tolerante à sombra; Ss/sistemas sexuais: (1) hermafrodita, (2) monóica, (3) dióica; Lo/locais de ocorrência: (1) mata mesófila, (2) mata de galeria, (3) cerradão, (4) matas mesófila/galeria, (5) matas mesófila/cerradão, (6) matas galeria/ cerradão e (7) matas mesófila/galeria/cerradão; Ps/classes de peso de sementes: (1) S $\leq 0,1 \mathrm{~g}$; (2) $0,1 \mathrm{~g}<\mathrm{S}<1,5 \mathrm{~g}$; (3) P $\geq 1,5 \mathrm{~g}$; In: Informação obtida através de revisão bibliográfica para a espécie (1) e gênero (2). Informação obtida através de observação direta para a espécie (3) e gênero (4).

tomentosum não se adequou perfeitamente. Nesta espécie, os cotilédones foliáceos fotossintéticos são erguidos bem acima do nível do solo, mas não pelo desenvolvimento do hipocótilo e sim pelo desenvolvimento dos pecíolos cotiledonares que se desenvolvem a partir do colo da plântula, ou seja, no nível do solo. Como esta espécie possui uma estrutura funcionalmente semelhante às plântulas fanero-epígeofoliáceas, ela foi considerada como do tipo PEF.

Os resultados demonstram a adequação da classificação, mesmo sendo simples e focada somente em características cotiledonares. Entretanto, é preciso relembrar que o desenvolvimento e o estabelecimento são processos dinâmicos e o uso de uma classificação baseada somente em um estágio de vida destas espécies pode ocultar informações importantes sobre as funções da morfologia das plantas.

$\mathrm{Na}$ análise da distribuição dos tipos morfofuncionais (figura 1), o tipo PEF foi significativamente mais freqüente $(51 \%)$, CHR $(23 \%)$ e PER $(17 \%)$ foram intermediários, e PHR ( $8 \%)$ e CER $(1 \%)$ foram significativamente menos comuns $\left(\chi^{2}=89,9 ; p<0,001\right)$. Pôde-se perceber uma distribuição semelhante a outras quatro florestas tropicais já estudadas (tabela 2), nas quais o tipo morfofuncional PEF foi sempre o mais freqüente, variando de $39 \%$ a $51 \%$ do total de espécies, enquanto o tipo morfofuncional CER foi o mais raro, variando de $1 \%$ a $8 \%$. A similaridade na abundância 


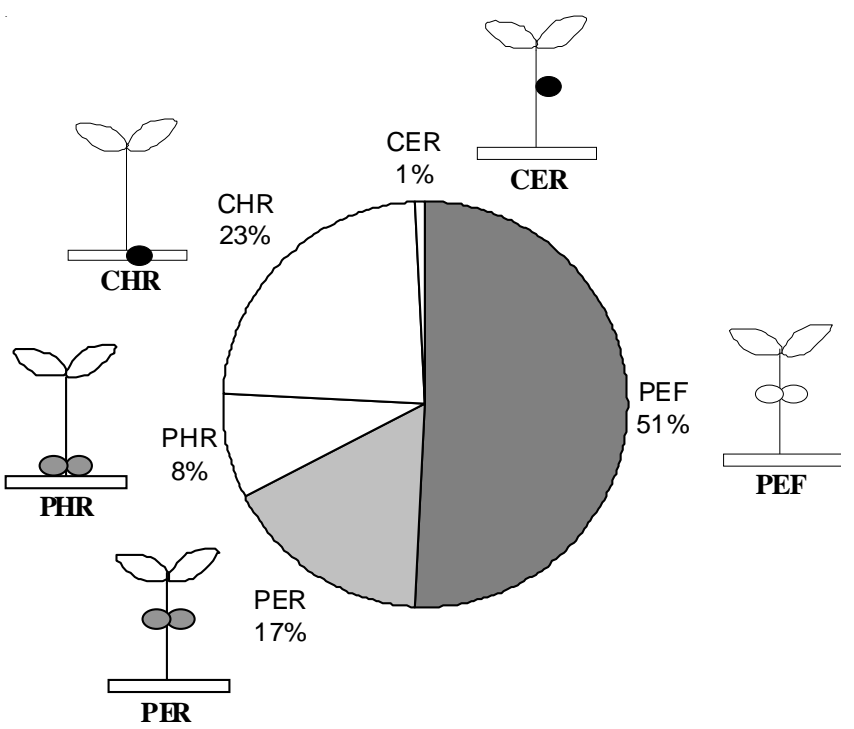

Figura 1. Distribuição dos tipos morfofuncionais das plântulas das espécies arbóreas da Estação Ecológica do Panga, Uberlândia, MG.

Figure 1. Functional morphology types of woody species of the Panga Ecological Station, Uberlândia, MG.

dos tipos morfofuncionais de plântulas entre as florestas tropicais comparadas sugere que pressões seletivas semelhantes podem estar agindo na determinação da morfologia das plântulas e que, se isto for correto, estudos de ecologia morfofuncional poderiam ser aplicados para os trópicos como um todo (Garwood 1995).

Grupos sucessionais em relação aos tipos morfofuncionais - A distribuição dos tipos morfofuncionais entre grupos sucessionais apresentou
Tabela 2. Distribuição porcentual dos tipos morfofuncionais de plântulas em cinco florestas tropicais.

Table 2. Percent distribution of seedling functional morphology types in five tropical forests.

\begin{tabular}{lrrllll}
\hline Estudos & $N^{\circ}$ spp & PEF PER PHR CHR CER \\
\hline Ng (1978) (Malásia) & 300 & $64^{*}$ & 10 & 18 & 8 \\
Garwood (1983) (Panamá) & 206 & 43 & 16 & \multicolumn{4}{l}{$41^{*}$} & \\
Rousteau (1983) (Guadalupe) & 102 & 51 & 16 & $31^{*}$ & 2 \\
Miquel (1987)(Gabão) & 210 & 39 & 25 & 9 & 22 & 5 \\
Presente Estudo (Brasil) & 122 & 51 & 17 & 8 & 23 & 1 \\
\hline
\end{tabular}

Fonte: Adaptado de Hladik \& Miquel (1990)

*PEF+ PER; *PHR + CHR + CER e • PHR + CHR

uma alta porcentagem do tipo PEF para as espécies pioneiras (75\%) e clímax exigentes de luz (55\%), o que corresponde à estratégia esperada para espécies em estágios sucessionais iniciais (figura 2). Esta relação foi significativa $\left(\chi^{2}=34,01 ; p<0,001\right)$. Esses grupos sucessionais, principalmente as pioneiras, tendem a crescer rapidamente para competir por espaço e luz (Ng 1978). Neste caso, cotilédones prontamente fotossintetizantes, significam uma vantagem adicional.

Por outro lado, uma alta porcentagem do tipo morfofuncional CHR foi observada para as espécies clímax tolerantes à sombra $(52 \%)$. Geralmente estas espécies formam bancos de plântulas e podem sobreviver no sub-bosque por longos períodos (Schiavini et al. 2001). Para isso é fundamental que possuam substâncias nutritivas armazenadas e o tipo CHR mantém estas reservas relativamente protegidas. Outra
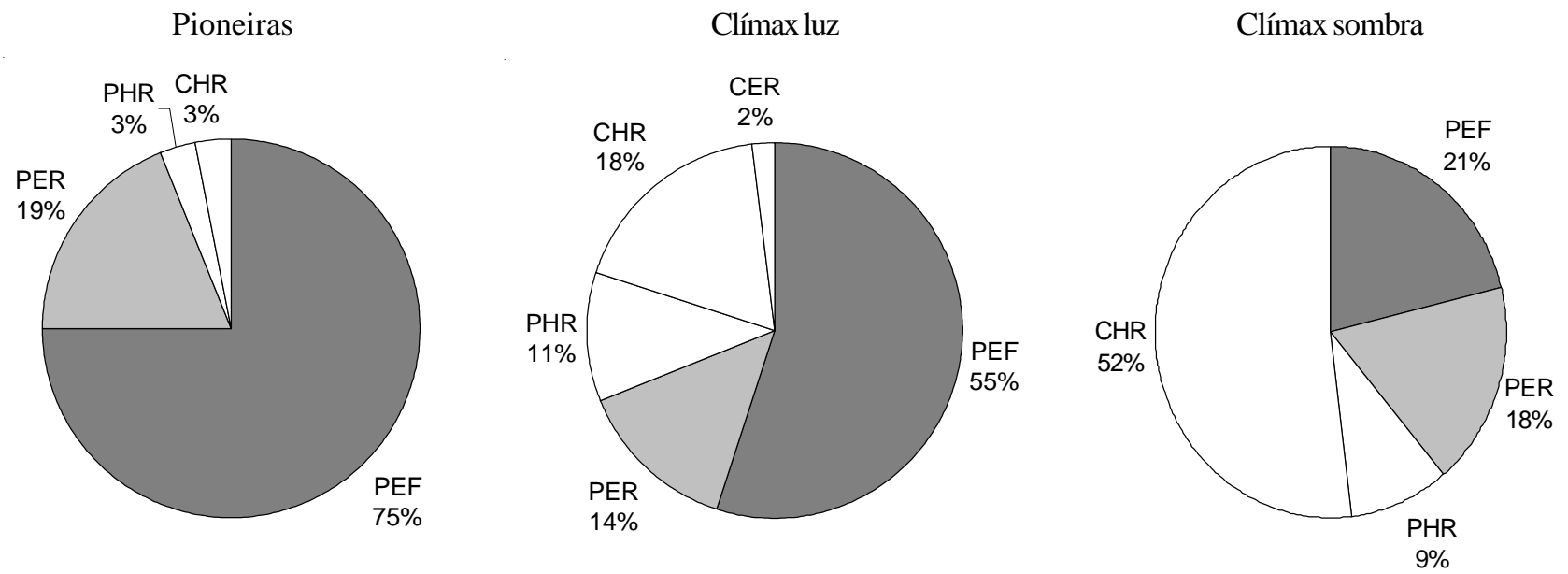

Figura 2. Distribuição porcentual dos tipos morfofuncionais de plântulas das espécies arbóreas da Estação Ecológica do Panga, em grupos sucessionais.

Figure 2. Percent distribution of seedling functional morphology types of woody species of the Panga Ecological Station among successional groups. 
característica importante dos criptocotilédones é que o tegumento da semente pode funcionar como uma barreira protetora.

Os tipos morfofuncionais PER e PHR estão relativamente bem distribuídos nos três estágios sucessionais. Isto pode ser explicado pela subdivisão dos cotilédones armazenadores em fotossintetizantes ou não. Apesar dos dados mostrarem esta tendência, não foi possível testar esta hipótese devido ao número insuficiente de espécies representando estas categorias. Outro problema em relação ao tamanho de amostra ocorreu com o tipo morfofuncional CER. Não foi possível realizar nenhuma consideração, uma vez que só a espécie Virola sebifera apresentou este tipo morfofuncional.

Peso das sementes em relação aos tipos morfofuncionais - Cerca de $73 \%$ das sementes mais leves $(\mathrm{S} \leq 0,1 \mathrm{~g})$ corresponderam ao tipo morfofuncional PEF (figura 3). O tipo PEF é freqüente em espécies de estágios sucessionais iniciais, as quais investem em uma grande quantidade de sementes de pouca biomassa, ou seja, com pouca ou nenhuma capacidade de armazenamento. Por outro lado, a maioria das espécies com sementes mais pesadas $(\geq 1,5 \mathrm{~g})$ apresentou plântulas do tipo CHR, com um predomínio de espécies de estágios finais de sucessão. Estas espécies tardias investem em poucas

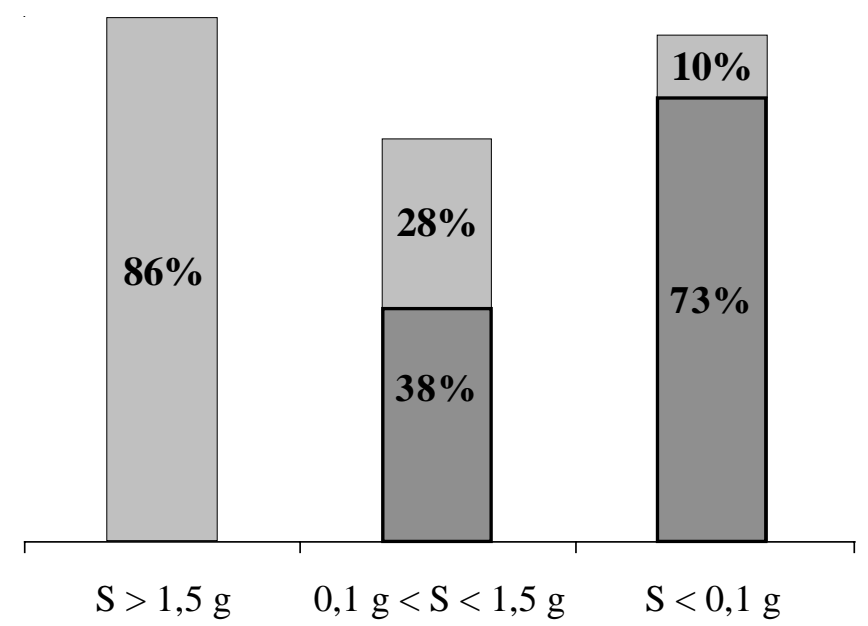

Figura 3. Distribuição porcentual das plântulas fanero-epígeofoliáceas (PEF) e cripto-hipógeo-armazenadoras (CHR) em três classes de peso de sementes (g) para espécies arbóreas da Estação Ecológica do Panga, Uberlândia, MG. $\square=$ PEF, $=$ CHR.

Figure 3. Percent distribution of phanero-epigeal-foliaceous (PEF) and crypto-hypogeal-reserve (CHR) seedling types among three classes of seed weight $(\mathrm{g})$ in woody species of the Panga Ecological Station, Uberlândia, MG. $\square=\mathrm{PEF}, \quad=\mathrm{CHR}$. sementes, porém ricas em reservas nutritivas, as quais conferem às sementes uma maior biomassa.

Os resultados obtidos na EEP são semelhantes àqueles encontrados na Malásia (Ng 1978) e no Gabão (Miquel 1987), apesar deles terem utilizado o tamanho das sementes em vez do peso. No presente trabalho, utilizou-se o peso das sementes pois, acredita-se que esta medida está mais correlacionada com o investimento da planta parental do que o tamanho, característica esta que pode ser influenciada pela forma de dispersão.

$\mathrm{Ng}$ (1978) mostrou, para as espécies arbóreas da Malásia, que a abundância relativa de plântulas faneroepígeas (PEF + PER) diminuía com o aumento do tamanho da semente, de $100 \%$ para sementes menores que $3 \mathrm{~mm}$ para $50 \%$ em sementes maiores que $20 \mathrm{~mm}$. Resultados semelhantes foram obtidos para plântulas no Gabão (Hladik \& Miquel 1990), onde apenas 5\% das espécies com sementes $\geq 40 \mathrm{~mm}$ possuíam plântulas do tipo PEF, com esta porcentagem aumentando para $19 \%$ nas sementes menores $(20 \leq \mathrm{S}<40 \mathrm{~mm})$.

Sistemas sexuais em relação aos tipos morfofuncionais - Ao classificar os tipos morfofuncionais de plântulas em hermafroditas, monóicos e dióicos, observou-se que a maior parte das espécies é hermafrodita e esta categoria está bem distribuída dentre os cinco tipos morfofuncionais de plântulas. No entanto, entre as plantas dióicas observou-se uma alta porcentagem (41\%) de espécies com plântulas do tipo CHR (figura 4). Esta associação entre dioicia e plântulas CHR é provavelmente indireta, apesar de Vogel (1980) sugerir que este tipo morfofuncional permitiria uma maior capacidade de sobrevivência a estas plântulas em condições de mata, o que seria vital para espécies dióicas. Ele argumenta que a continuação das populações de plantas dióicas depende de árvores matrizes isoladas, requerendo plântulas com maior capacidade de sobrevivência.

Analisando os tipos morfofuncionais segundo sua vulnerabilidade, Vogel (1980) concluiu que plântulas do tipo CHR seriam mais resistentes, por possuírem suas reservas nutritivas protegidas pelo tegumento da semente e por estarem sob o solo. É possível, no entanto, que a associação entre dioicia, hábito lenhoso, estágios secundários de sucessão e dispersão por animais (IbarraManríquez \& Oyama 1992), todos fatores associados com plântulas CHR, seja mais importante que uma relação direta entre dioicia e esta estratégia de estabelecimento.

Alocação de biomassa em relação aos tipos morfofuncionais - Segundo os dados de grupos 

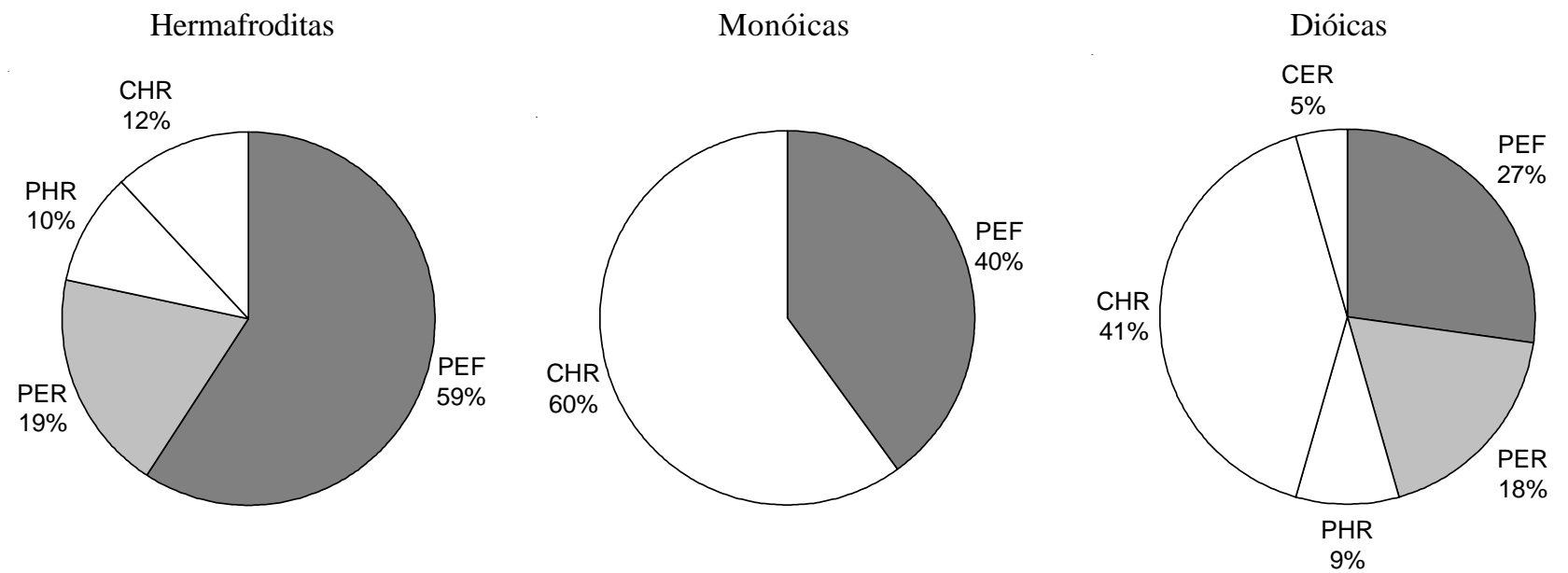

Figura 4. Distribuição porcentual dos tipos morfofuncionais de plântulas das espécies arbóreas da Estação Ecológica do Panga, Uberlândia, Minas Gerais, em função do tipo de sistema sexual.

Figure 4. Percent distribution of seedling functional morphology types of woody species of the "Panga" Ecological Station, Uberlândia, Minas Gerais, with different sexual systems.

sucessionais e peso de sementes já analisados, pôde-se observar que existe uma tendência para que plântulas PEF sejam pequenas, geradas por sementes pequenas, com pouca ou nenhuma reserva nutritiva, enquanto que plântulas CHR tendem a ser grandes, geradas por sementes grandes e com considerável reserva nutritiva.

Portanto, a partir destas relações seria plausível especular que a alocação de biomassa poderia ser diferente para os diferentes tipos morfofuncionais. Para verificar possíveis correlações entre a morfologia funcional das plântulas e a alocação diferencial de sua biomassa para raiz ou parte aérea, 27 espécies arbóreas foram analisadas (tabela 3), mas a análise não mostrou nenhuma diferença significativa na relação entre a biomassa de raiz e parte aérea entre os tipos morfofuncionais $(F=0,459 ; p=0,714)$. Como este resultado pode ter sido influenciado pelo número de espécies analisadas em cada tipo, sugere-se que novas análises sejam realizadas.

Os resultados tampouco foram significativos quanto a possíveis diferenças na alocação de biomassa raiz/ parte aérea entre espécies das diferentes fitocenoses estudadas $(F=2,106 ; p=0,116)$. Espécies exclusivas do cerradão e presentes no grupo cerradão/mata mesófila semidecídua, parecem apresentar as maiores taxas de alocação de biomassa na parte radicular, mas a variação foi grande e o número de espécies analisado foi restrito. Espécies características destas áreas são espécies típicas do cerrado e tendem a desenvolver sistemas radiculares mais profundos e/ou espessados, em busca de camadas mais úmidas do solo, de maneira a sobreviver à seca e às queimadas ocasionais destas áreas (Moreira \& Klink 2000).

Estes resultados sugerem que mesmo que as plântulas apresentem diferentes padrões de alocação de biomassa entre os ambientes, estes padrões não terão relação direta com os tipos morfofuncionais. Eles provavelmente vão variar com as exigências do ambiente e condições de disponibilidade de luz e umidade para a continuação do desenvolvimento inicial (Moreira \& Klink 2000, Hoffmann \& Franco 2003).

As espécies vicariantes podem ser um bom exemplo desta flexibilidade da forma de alocação. Em plantas congenéricas a morfologia funcional das plântulas é geralmente a mesma. No entanto, plantas de cerrado apresentam um maior espessamento radicular, enquanto que plantas de matas podem apresentar uma maior alocação de biomassa na parte aérea e um espessamento radicular menor (Hoffmann \& Franco 2003). Por exemplo, podemos citar as três espécies do gênero Qualea. Q. dichotoma, presente nas três fitocenoses, Q. grandiflora, presente na mata mesófila semidecídua e cerradão e $Q$. multiflora, presente apenas no cerradão, são espécies congenéricas e possuem plântulas do mesmo tipo morfofuncional, mas diferem sob o aspecto de alocação de biomassa (tabela 3).

Aparentemente, as funções da morfologia das plântulas no processo de estabelecimento limitam-se ao primeiro estágio de desenvolvimento, participando efetivamente no aumento das suas chances de sobrevivência. A partir do momento em que a plântula sobrevive ao estágio inicial de estabelecimento, novas 
Tabela 3. Valores de alocação de biomassa raiz/parte aérea (R/A) e seus respectivos locais de ocorrência (LO) para 27 espécies arbóreas da Estação Ecológica do Panga, Uberlândia, MG. Locais de Ocorrência: 1 = mata mesófila, 2 = mata de galeria, 3 = cerradão, 4 = matas mesófila/galeria, $5=$ matas mesófila/cerradão, $6=$ matas galeria/cerradão e $7=$ todos os ambientes.

Table 3. Biomass allocation ratio, root/stem (R/A), and site of occurrence for 27 woody species of the Panga Ecological Station, Uberlândia, MG. Site of occurrence (LO): 1 = semideciduous forest, 2 = gallery forest, 3 = cerradão (dense cerrado woodland), $4=$ semideciduous forest/gallery forest, $5=$ semideciduous forest/cerradão, $6=$ gallery forest/cerradão, and $7=$ all habitats.

\begin{tabular}{|c|c|c|c|}
\hline Espécie & Tipo de Plântula & $\mathrm{R} / \mathrm{A}$ & LO \\
\hline Alibertia sessilis (Vell.) K. Schum. & PEF & 0,19 & 7 \\
\hline Calyptranthes widgreniana $\mathrm{O}$. Berg & PEF & 0,32 & 2 \\
\hline Cariniana estrellensis (Raddi) Kuntze & PEF & 1,07 & 1 \\
\hline Coussarea hydrangeaefolia (Benth.) Benth. \& Hook. & PEF & 2,15 & 7 \\
\hline Ixora gardneriana Benth. & PEF & 0,54 & 2 \\
\hline Kielmeyera coriacea Mart. & PEF & 2,42 & 3 \\
\hline Lithrea molleoides Engl. & PEF & 0,30 & 7 \\
\hline Myrcia rostrata DC. & PEF & 1,33 & 5 \\
\hline Qualea dichotoma (Mart.) Warm. & PEF & 0,48 & 7 \\
\hline Qualea grandiflora Mart. & PEF & 7,66 & 5 \\
\hline Qualea multiflora Mart. & $\mathrm{PEF}$ & 4,84 & 3 \\
\hline Senna silvestris (Vell.) H.S. Irwin \& Barneby & PEF & 0,20 & 3 \\
\hline Styrax camporum Pohl & PEF & 0,29 & 7 \\
\hline Vochysia tucanorum Mart. & $\mathrm{PEF}$ & 0,40 & 7 \\
\hline Copaifera langsdorffii Desf. & PER & 0,60 & 7 \\
\hline Tabebuia impetiginosa (Mart. ex DC.) Standl. & PER & 2,09 & 5 \\
\hline Anadenanthera macrocarpa (Benth.) Brenan & PHR & 0,45 & 7 \\
\hline Connarus suberosus Planch & PHR & 2,51 & 3 \\
\hline Inga affinis DC. & PHR & 0,61 & 4 \\
\hline Pouteria gardneri (Mart. \& Miq.) Baehni & PHR & 0,76 & 5 \\
\hline Calophyllum brasiliense Cambess. & CHR & 0,58 & 2 \\
\hline Cheiloclinium cognatum (Miers) A.C. Sm. & CHR & 0,83 & 4 \\
\hline Cupania vernalis Cambess. & CHR & 0,95 & 5 \\
\hline Matayba guianensis Aubl. & $\mathrm{CHR}$ & 0,35 & 7 \\
\hline Nectandra cissiflora Nees & CHR & 0,57 & 4 \\
\hline Platypodium elegans Vogel & CHR & 0,42 & 7 \\
\hline Virola sebifera Aubl. & CER & 0,85 & 5 \\
\hline
\end{tabular}

características morfológicas se desenvolvem e a plântula passa a depender diretamente do meio em que se estabeleceu. Isto pode ser demonstrado pela independência da alocação da biomassa e das estratégias de crescimento em relação às características morfológicas iniciais das plântulas.

Locais de ocorrência em relação aos tipos morfofuncionais de plântulas - Alguns trabalhos (Vogel 1980, Lima 1989) sugerem que os tipos morfofuncionais de plântulas estariam distribuídos em microhabitats específicos. Por exemplo, Lima (1989) sugere que os tipos morfofuncionais fanero-epígeos (PEF + PER) seriam mais abundantes em matas secas com alta disponibilidade de luz e em florestas não inundáveis. Já o tipo morfofuncional CHR estaria mais relacionado à formações vegetais periodicamente alagáveis ou a formações de situações edáficas e/ou climáticas adversas como aquelas encontradas no cerrado sensu stricto.

Analisando a distribuição das espécies arbóreas da Estação Ecológica do Panga em: 1) espécies exclusivas da mata mesófila semidecídua, 2) espécies presentes na mata de galeria e 3 ) espécies presentes no cerradão, observou-se que as espécies com plântulas faneroepígeas (PEF + PER) encontraram-se distribuídas principalmente no cerradão e na mata mesófila semidecídua (figura 5). Estas fitocenoses são consideradas relativamente secas e sem períodos de inundação, além da maior incidência de luz devida à deciduidade parcial de ambas as fitocenoses. Em relação 


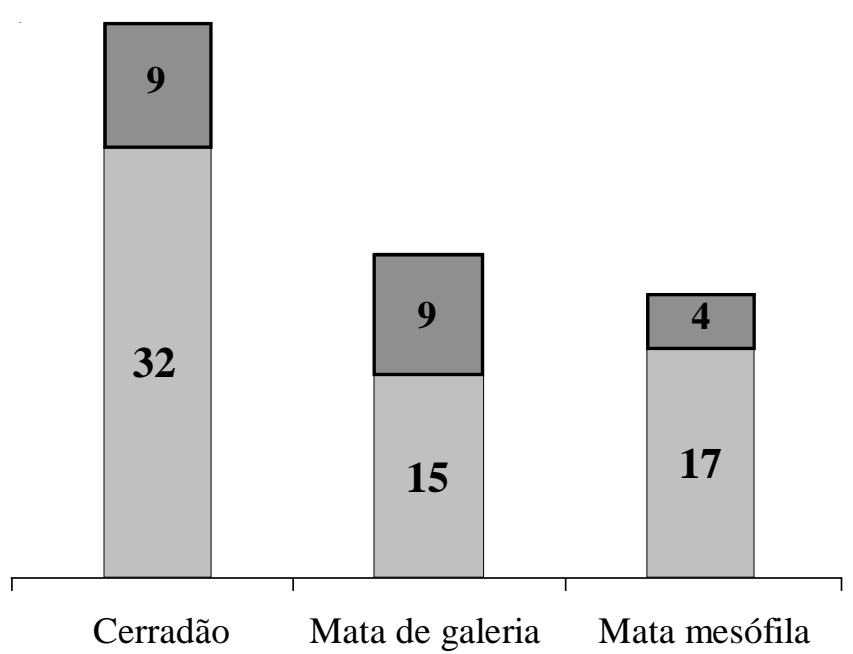

Figura 5. Distribuição dos tipos morfofuncionais faneroepígeos (PEF + PER) e cripto-hipógeo-armazenadores (CHR) na Estação Ecológica do Panga, Uberlândia, Minas Gerais, em valores absolutos, na diferentes fisionomias de vegetação. As espécies arbóreas foram separadas em exclusivas da mata mesófila semidecídua, ocorrentes na mata de galeria e ocorrentes no cerradão. $\square=\mathrm{PEF}+\mathrm{PER}, \square=\mathrm{CHR}$.

Figure 5. Distribution of phanero-epigeal (PEF+PER) and crypto-hypogeal-reserve (CHR) seedling types in woody species of the "Panga" Ecological Station, Uberlândia, Minas Gerais in absolute values. The species were separated in exclusive of semideciduous forest, occurring in the gallery forest and occurring in the cerradão areas. $=$ PEF + PER, $=\mathrm{CHR}$.

às espécies com plântulas do tipo CHR, pôde-se observar uma porcentagem considerável destas espécies na mata de galeria e no cerradão. Este resultado corresponderia à hipótese de Lima (1989), uma vez que a mata de galeria estudada possui períodos de inundação, enquanto que o Cerradão, tem solos bem drenados, mas tão pobres quanto os do cerrado contíguo.

Entretanto, é necessário salientar que a diferença de amostragem entre as três fitocenoses contínuas pode estar influenciando estes resultados. Durante o levantamento fitossociológico foram demarcados oito transectos, que resultaram em 21 parcelas na mata de galeria, 150 na mata mesófila semidecídua e 40 no cerradão. Além disso, uma melhor análise seria possível caso o número de espécies exclusivas a cada uma das fitocenoses fosse maior.

Síndromes de dispersão e épocas de frutificação em relação aos tipos morfofuncionais - Ao se dividirem as espécies arbóreas da Estação Ecológica do Panga em zoocóricas, anemocóricas e autocóricas, pôde-se observar que as três síndromes de dispersão estão distribuídas de forma similar dentre os tipos morfofuncionais (tabela 4). Hladik \& Miquel (1990) relacionaram os grupos morfofuncionais com alguns grupos específicos de vertebrados, encontrando correlações, tais como, um maior número de espécies com plântulas do tipo CHR dispersas por macacos. A dispersão deste tipo morfo-funcional, que está associado a sementes maiores, deve ser facilitado por vetores de maior porte.

Em relação às épocas de frutificação, pôde-se observar que as espécies arbóreas que apresentavam plântulas PEF e PER frutificaram principalmente nos meses mais secos (57\% e $72 \%$, respectivamente), ao contrário das espécies com plântulas cripto-hipógeas (CHR), que frutificaram principalmente na estação chuvosa (62\%). Existem sugestões na literatura que na estação chuvosa a atividade de vetores bióticos, associados às sementes maiores do tipo CHR, seria mais intensa (e.g. Gottsberger \& Silberbauer-Gotsberger 1983). Para obtenção de resultados mais conclusivos seria necessário utilizar como referência os períodos específicos de dispersão das sementes.

A morfologia funcional das plântulas na área estudada parece estar relacionada, como em outras florestas tropicais, principalmente com o estágio sucessional. Plantas clímax tardias têm sementes preponderantemente CHR, que exibem maior capacidade de armazenamento e proteção durante o processo de germinação e estabelecimento. No outro extremo, plântulas de espécies pioneiras têm cotilédones expostos e fotossintetizantes (PEF), que rapidamente assumem a função de nutrir a plântula em desenvolvimento. Fatores como tipo de dispersão, tamanho das sementes e até mesmo sistema sexual, parecem estar indiretamente associados à morfologia

Tabela 4. Distribuição porcentual dos tipos morfológicos de plântulas de acordo com os tipos de dispersão para as espécies arbóreas da Estação Ecológica do Panga, Uberlândia, MG.

Table 4. Seedling functional morphology types and dispersal modes of woody species of the "Panga" Ecological Station, Uberlândia, MG.

\begin{tabular}{lccc}
\hline & Zoocoria & Anemocoria & Autocoria \\
\hline PEF & $60 \%$ & $32 \%$ & $8 \%$ \\
PER & $45 \%$ & $40 \%$ & $15 \%$ \\
PHR & $60 \%$ & $30 \%$ & $10 \%$ \\
CHR & $96 \%$ & $4 \%$ & - \\
\hline
\end{tabular}


funcional apropriada a cada estágio sucessional. Após o processo inicial de estabelecimento, a morfologia funcional tem pouco efeito, e as condições ambientais e exigências específicas ao crescimento, em determinados habitats, tomam o controle do processo de estabelecimento. Estratégias de alocação de recursos ao longo dos primeiros meses de crescimento, por exemplo, parecem ser independentes do tipo de morfologia funcional. Apesar de provavelmente limitada a fase inicial do estabelecimento, a influência da morfologia funcional das plântulas no processo de estabelecimento parece ser importante e comum às várias florestas tropicais já estudadas. A classificação utilizada parece se adequar às plântulas destas espécies lenhosas do bioma cerrado e, a morfologia das plântulas, pode ser utilizada para entender o processo de regeneração nestas florestas e orientar a escolha de espécies para projetos de recuperação.

Agradecimentos - O trabalho foi parte do Mestrado em Engenharia Florestal da primeira autora, realizado no Departamento de Ciências Florestais da Universidade Federal de Lavras, que também proveu condições para algumas das análises. A autora foi bolsista da Fapemig e Capes. O Instituto de Biologia da UFU possibilitou infra-estrutura para o restante das análises e facilidades para o trabalho de campo. Parte do trabalho de campo e apoio logístico complementar foram feitos no âmbito do projeto integrado do CNPq (ref. 520872 / 96-7).

\section{Referências bibliográficas}

AGUIAR, I.B. \& PIÑA-RODRIGUES, F.C.M. 1993. Maturação e dispersão de sementes. In Sementes florestais tropicais (I.B. Aguiar, F.C.M. Piña-Rodrigues \& A.M.B. Figliolia, eds.). Associação Brasileira de Tecnologia de Sementes, Brasília, p.215-274.

DUKE, J.A. 1965. Keys for the identification of seedlings of some prominent woody species in eight forest types in Puerto Rico. Annals of the Missouri Botanical Garden 52:314-350.

DUKE, J.A. 1969. On tropical tree seedlings: I. Seeds, seedlings, systems and systematics. Annals of the Missouri Botanical Garden 56:125-161.

GARWWOD, N.C. 1983. Seed germination in a seasonal tropical forest in Panama: a community study. Ecological Monographs 53:159-181.

GARWOOD, N.C. 1995. Functional morphology of tropical tree seedlings. In The ecology of tropical forest tree seedlings (M.D. Swaine, ed.). The Parthenon Publishing Group, New York, p.59-129.

GOTTSBERGER, G. \& SILBERBAUER-GOTTSBERGER, I. 1983. Dispersal and distribution in the cerrado vegetation of Brazil. Sonderbänd des Naturwissenschaftlichen Vereins in Hamburg 7:315-352.
HOFFMANN, W.A. \& FRANCO, A.C. 2003. Comparative growth analysis of tropical forest and savanna woody plants using phylogenetically independent contrasts. Journal of Ecology 91: 475-484

HOWE, H.F. \& WESTLEY, L.C. 1997. Ecology of pollination and seed dispersal. In Plant Ecology (M.J. Crawley, ed.). Blackweell Scientific Publication, Oxford, p.262-283.

HOWE, H.F. \& SMALLWOOD, J. 1982. Ecology of seed dispersal. Annual Review of Ecology Systematics 13:201-228.

HLADIK, A. \& MIQUEL, S. 1990. Seedlings types and plant establishment in an African rain forest. In Reproductive ecology of tropical forest plants (K.S. Bawa \& M. Hadley, eds.). Unesco/Parthenon, Paris/Carnforth, p.261-282.

IBARRA-MANRÍQUEZ, G. \& OYAMA, K. 1992. Ecological correlates of reproductive traits of Mexican rain forest trees. American Journal of Botany 79:383-394.

IBARRA-MANRÍQUEZ, G., RAMOS, M.M. \& OYAMA, K. 2001. Seedling functional types in a lowland rain forest in Mexico. American Journal of Botany 88:1801-1812.

LIMA, H.C. 1989. Tribo Dalbergiae (Leguminosae, Papilionoideae): morfologia dos frutos, sementes e plântulas e sua aplicação na sistemática. Arquivos do Jardim Botânico do Rio de Janeiro 30:1-42.

MARQUES, M.C.M. 2002. Dinâmica da dispersão de sementes e regeneração de plantas da planície litorânea da Ilha do Mel, PR. Tese de doutorado, Universidade Estadual de Campinas, Campinas.

MIQUEL, S. 1987. Morphologie fonctionnele de plantules d'espèces forestières du Gabon. Bulletin du Muséum National d'Histoire Naturelle 9:101-121.

MOREIRA, A.G. \& KLINK, C.A. 2000. Biomass allocation and growth of tree species seedlings from two contrasting Brazilian savannas. Ecotropicos 13:43-51.

MORENO, M.I.C. \& SCHIAVINI, I. 2001. Relação entre vegetação e solo em um gradiente florestal na Estação Ecológica do Panga, Uberlândia (MG). Revista Brasileira de Botânica 24(supl.):537-544.

NG, F.S.P. 1978. Strategies of establishment in Malayan forest trees. In Tropical trees as living systems (P.B.P. Tomlinson \& M.H. Zimmermann, eds.). Cambridge University Press, London, p.129-162.

OLIVEIRA, P.E.A.M. \& PAULA, F.R. 2001. Fenologia e Biologia reprodutiva de plantas de matas do Brasil central. In Cerrado: caracterizações e recuperação de matas de galeria (J.F. Ribeiro, C.E.L. Fonseca \& J.C. Souza-Silva, eds.). Embrapa-CPAC, Brasília, p.303-332.

PIRES-O'BRIEN, M. J. \& O'BRIEN, C.M. 1995. Ecologia e modelamento de florestas tropicais, Faculdade de Ciências Agrárias do Pará, Belém.

ROSA, R., LIMA, S.C. \& ASSUNÇÃO, W.L. 1991. Abordagem preliminar das condições climáticas de Uberlândia (MG). Revista Sociedade e Natureza 3:91-108.

ROUSTEAU, A. 1983. 100 plantules d'arbes guadeloupéens. Aspects morphologiques et écologiques. 3ème cycle Thèses, Université Pierre \& Marie Curie, Paris. 
SCHIAVINI, I. \& ARAÚJO, G.M. 1989. Considerações sobre a vegetação da Reserva Ecológica do Panga (Uberlândia). Sociedade e Natureza 1:61-66.

SCHIAVINI, I., RESENDE, J.C.F. \& AQUINO, F.G. 2001. Dinâmica de populações de espécies arbóreas em mata de galeria e mata mesófila na margem do Ribeirão do Panga, MG. In Cerrado: Caracterização e Recuperação de Matas de Galeria (J.F. Ribeiro, C.E.L. Fonseca \& J.C. Sousa-Silva, eds.). Embrapa-CPAC, Brasília, p.267-299.
SOKAL, R.R. \& ROHLF, J.E. 1981. Biometry. Freeman, San Francisco.

SWAINE, M.D. \& WHITMORE, T.C. 1988. On the definition of ecological species groups in tropical rain forests. Vegetatio 75:81-86.

VOGEL, E.F. 1980. Seedlings of dicotyledons: structure, development, types: descriptions of 150 woody Malesian taxa. Centre for Publishing and Documentation, Wageningen. 